SHORT COMMUNICATION

\title{
Viscosity measurements of low temperature molten salts
}

\author{
FRANCIS M. DONAHUE, JOHN F. ROSS \\ Chemical Engineering Department, The University of Michigan, Ann Arbor, MI 48109-2136, USA
}

Received 12 June 1988

\author{
Nomenclature \\ $m$ consistency (units dependent upon value of flow \\ index)
}

\section{Introduction}

Low temperature molten salts are a new class of electrolytes for battery applications (e.g. [1]). Viscosity measurements of low temperature molten salts based on mixtures of aluminium or copper halide and alkylpyridinium or dialkylimidazolium halide have been reported [1-5]. They have shown strong dependences of viscosity with changes in composition in basic (i.e. organic-rich) melts $[1,2,4,5]$. Further, 'gaps' in the liquidus lines, i.e. regions where fusion does not occur, only a glass transition at very low temperatures, have been shown for binary melts containing 1-methyl-3-ethylimidazolium chloride (MEIC) [1] and bromide [2] as the organic salt. Finally, measurements of chemical shifts for proton and carbon nuclear magnetic resonance spectra of basic MEIC$\mathrm{AlCl}_{3}$ melts have been explained in terms of oligomeric structures [6, 7]. All of these measurements used viscometers based on variations of Cannon-Fenske devices $[1-3,5]$ or the falling ball method [4]. These methods do not provide shear stress-shear rate data, which could be used to determine whether the melts behave like non-Newtonian fluids. This paper reports viscosity measurements of $\mathrm{MEIC}-\mathrm{AlCl}_{3}$ binary melts using a plate and cone viscometer (in order to obtain shear stress-shear rate data) and compares the viscosities measured using this technique with those of previous investigators.

\section{Experimental details}

The organic salt was synthesized and purified similarly to the method of Wilkes and co-workers [8]. Aluminium chloride (Fluka puriss.) was sublimed from a mixture of $\mathrm{NaCl}$ and aluminium wire in an evacuated vessel at $170^{\circ} \mathrm{C}$ onto a 'cold finger' cooled by an external flow of air. The viscometer was a Brookfield Model LVTD Digital Viscometer with CP-40, CP-42, and CP-52 cones. Temperature control was maintained by recirculating water through an insulated tube from a Lauda Model TK-30 constant temperature bath to the jacketed sample holder of the viscometer. The viscosity measurements were carried out in a Vacuum Atmospheres Company dry box equipped with a 'Dri Train' having oxygen removal capabilities.

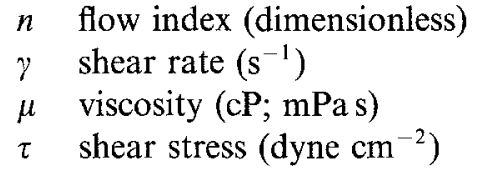

The dry box atmosphere was a mixture of purified helium and nitrogen. A summary of the experiments carried out is shown in Table 1.

\section{Results and discussion}

\subsection{Power law model and analysis}

In order to determine whether the melts exhibited Newtonian or non-Newtonian behavior, the shear stress-shear rate data were analyzed according to the power law equation (Ostwald-de Waele Model) [9], i.e.

$$
\tau=m \gamma^{n}
$$

where $\tau$ is the shear stress, $m$ is the consistency, $\gamma$ is the shear rate and $n$ is the flow index ( $n=1$ for Newtonian fluids and $n<1$ for pseudoplastic fluids). Values of consistency and flow index were obtained from log$\log$ plots of the shear stress vs the shear rate. The viscosity, $\mu$, was computed from

$$
\mu=m \gamma^{n-1}
$$

With the exceptions of the data from melts with a nominal $\mathrm{AlCl}_{3}$ mol fraction, $N$, of 0.35 at $30 \geqslant T$ $\left({ }^{\circ} \mathrm{C}\right) \geqslant 10$ and for $N=0.40$ at all temperatures indicated, the flow index was $1.00 \pm 0.02$, indicating Newtonian behavior.

\subsection{Non-Newtonian behavior}

The experiments which seemed to indicate nonNewtonian behavior were analyzed in order to compute the viscosity-shear rate relationship. The flow

Table 1. Summary of viscosity data

\begin{tabular}{lll}
\hline $\begin{array}{l}\text { Nominal } \\
\text { AlCl }\end{array}$ & $\begin{array}{l}\text { Temperature } \\
\text { mol fraction }\end{array}$ & $\begin{array}{l}\text { Number of } \\
\text { data pairs }\end{array}$ \\
\hline 0.31 & & \\
\hline 0.35 & $20-40$ & $5-7$ \\
0.40 & $10-35$ & $7-11$ \\
0.45 & $10-35$ & $8-10$ \\
0.64 & $0-25$ & $4-5$ \\
\hline
\end{tabular}

* In $5^{\circ} \mathrm{C}$ increments.

$\uparrow$ At each temperature 


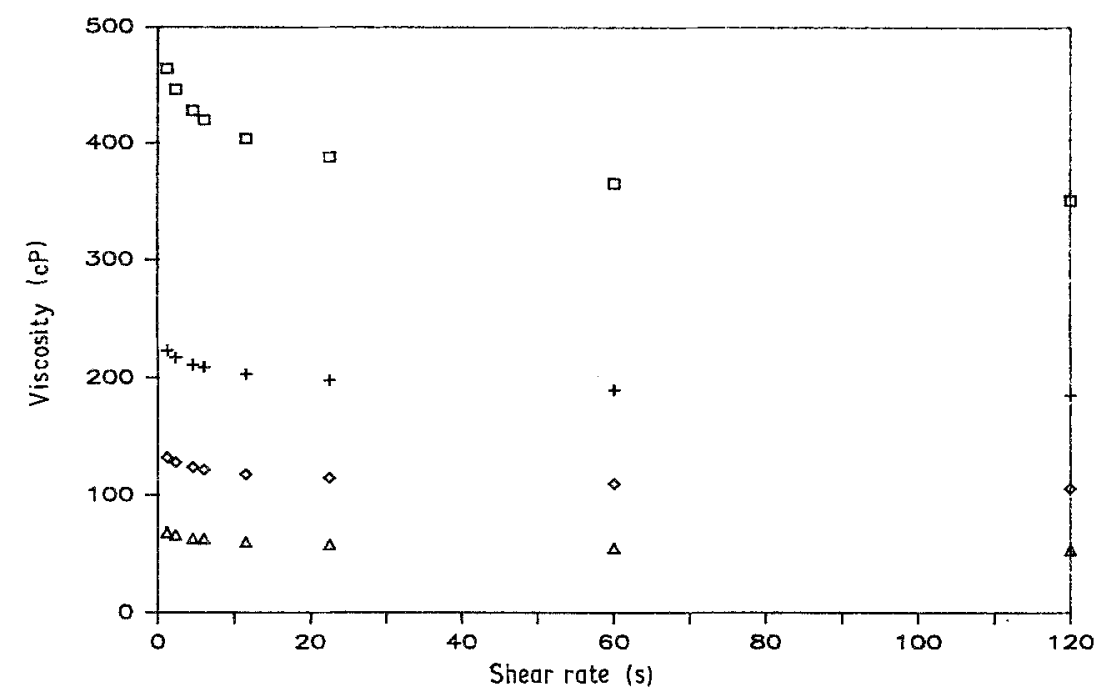

Fig. 1. Viscosity-shear rate behavior of melts with fiow indices less than unity. $N=0.35: \square, 10^{\circ} \mathrm{C} ;+, 20^{\circ} \mathrm{C} ; N=0.40: \diamond, 15^{\circ} \mathrm{C} ; \Delta, 30^{\circ} \mathrm{C}$.

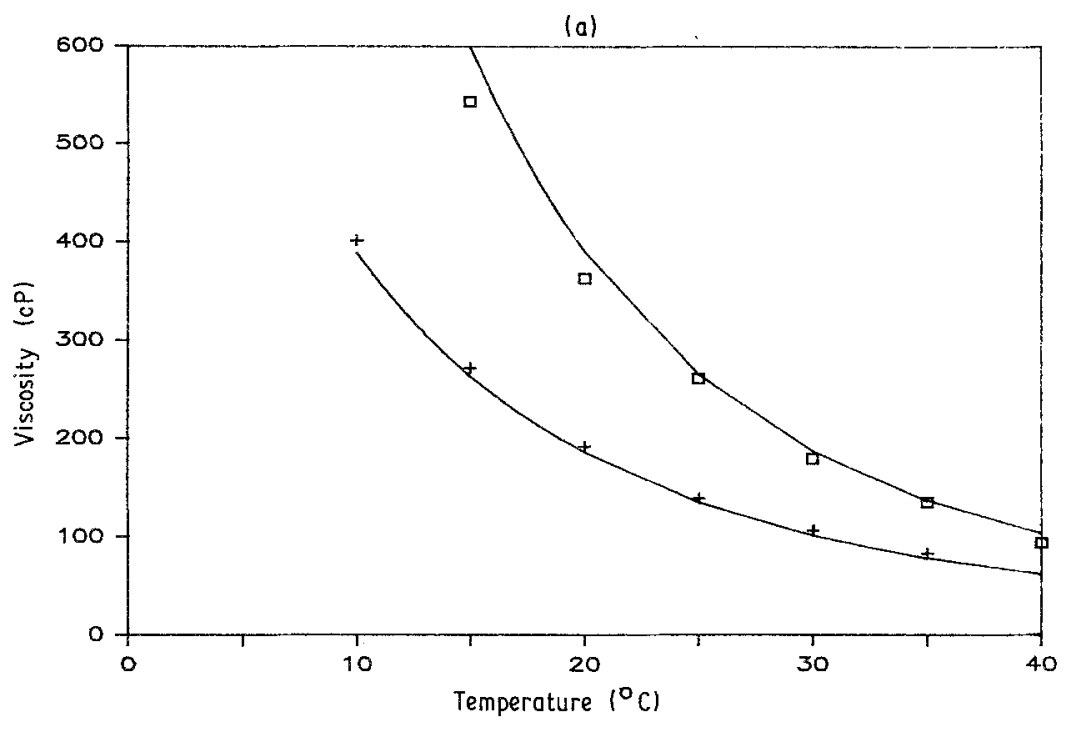

(b)

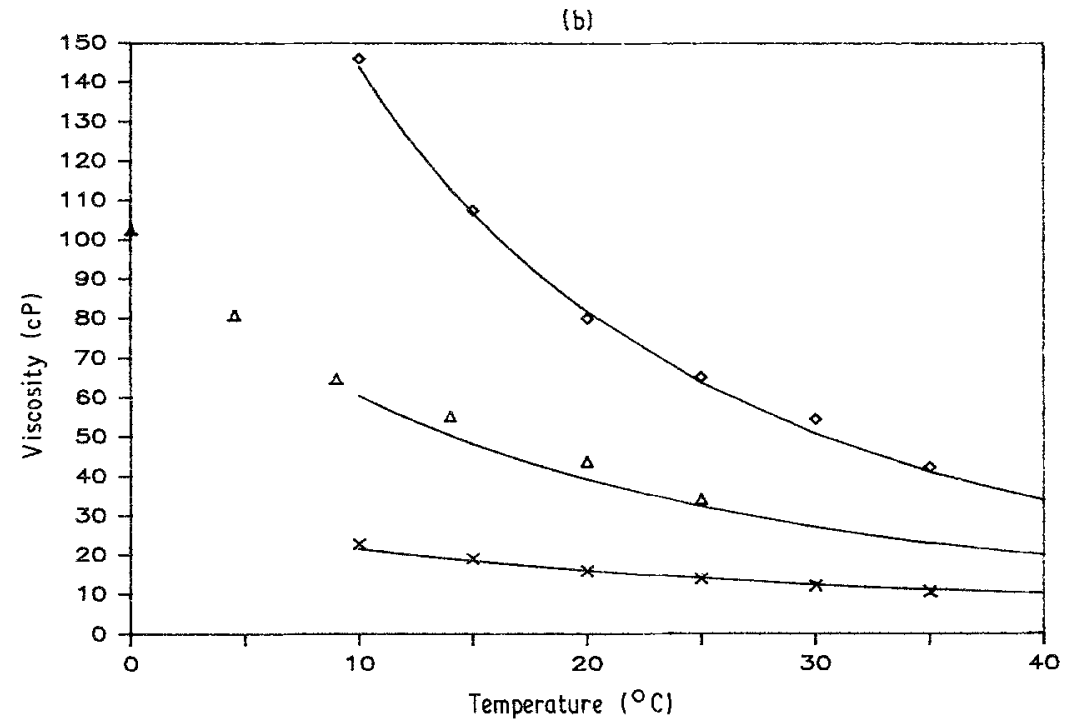

Fig. 2. Viscosity-temperature behavior of melts. (a) $\square, N=0.31 ;+, N=0.35 ;$ (b) $\diamond, N=0.40 ; \Delta, N=0.45 ; x, N=0.64$. 
Table 2. Computed activation energies ( $\left.k_{c a l m o l}{ }^{-1}\right)$

\begin{tabular}{ll}
\hline $\begin{array}{l}\text { Nominal } \mathrm{AlCl}_{3} \\
\text { mol fraction }\end{array}$ & $\begin{array}{l}\text { Activation } \\
\text { energy }\end{array}$ \\
\hline 0.31 & 12.4 \\
0.35 & 10.9 \\
0.40 & 8.4 \\
0.45 & 6.9 \\
0.64 & 5.3 \\
\hline
\end{tabular}

index was found to be equal to or greater than $0.90^{*}$, but always less than unity - indicating a slight dependence of the viscosity on the shear rate and slight pseudoplastic behavior. It is of interest that the melts which show this behavior are found in the vicinity of the liquidus 'gap' mentioned above. Figure 1 shows some of the data which exhibit this shear-thinning behavior.

\subsection{Viscosity computations and comparison}

Even the experiments which had flow indices less than unity (i.e. non-Newtonian behavior) exhibited linear dependencies of shear stress on shear rate for all practical purposes. In the absence of stronger evidence of non-Newtonian behavior, the viscosities for these melts (as well as those for which Newtonian behavior was observed) were computed from the linear shear stress-shear rate data. Figure 2 shows the computed values of viscosities from these experiments (symbols) while the curves show a correlation for viscosity developed by Fannin and co-workers for these binary melts based on their measurements [1]. The agreement between these data and the correlation indicates that the viscosity can be conveniently obtained for these (and similar) melts by either of the methods employed in the respective studies.

\subsection{Activation energies}

Unlike some studies on similar molten salts $[1,2]$, the viscosity data obtained in this study did provide an Arrhenius relationship over the entire temperature range. The computed activation energies are shown in
Table 2 . These values are very close to those computed by Fannin at $30^{\circ} \mathrm{C}$ [1], significantly larger than those reported for molten $\mathrm{HgCl}_{2}[10]$ and molten pyridinium salts [11, 12], similar to (but less than) those for $\mathrm{CuCl}$ MEIC binaries in basic melts [5], smaller than those for acidic CuCl-MEIC binaries where the viscosity rises steeply with increasing $\mathrm{CuCl}$ mol fractions [5] and approximately the same as those calculated by Carpio and co-workers for acidic alkylpyridinium chloride- $\mathrm{AlCl}_{3}$ binaries [3]. Although providing no quantitative information concerning melt structure, the magnitude of the activation energies, particularly in the more basic melts, indicates strong interactions among the components [1] - exceeding those of typical physical processes.

\section{Acknowledgements}

The authors wish to thank the Air Force Office of Scientific Research for financial support of this work under Grant No. AFOSR-85-0027. They would like to acknowledge the many fruitful discussions with Leif Simonsen and Russ Moy.

\section{References}

[1] A. A. Fannin, D. A. Floreani, L. A. King, J. S. Landers, B. J. Piersma, D. J. Stech, R. L. Vaughn, J. S. Wilkes and J. L. Williams, J. Phys. Chem. 88 (1984) 2614.

[2] J. R. Sanders, E. H. Ward and C. L. Hussey, J. Electrochem. Soc. 133 (1986) 325.

[3] R. Carpio, L. A. King, R. E. Lindstrom, J. C. Nardi and C. L. Hussey, ibid. 126 (1979).

[4] C. Nanjundiah, K. Shimizu and R. A. Osteryoung, ibid. 129 (1982) 2474.

[5] S. A. Bolkan and J. T. Yoke, J. Chem. Eng. Data 31 (1986) 194.

[6] A. A. Fannin, L. A. King, J. A. Leviskey and J. S. Wilkes, J. Phys. Chem. 88 (1984) 2609.

[7] J. S. Wilkes, C. L. Hussey and J. R. Sanders, Polyhedron 5 (1986) 1567.

[8] J. S. Wilkes, J. A. Leviskey, R. A. Wilson and C. L. Hussey, Inorg. Chem. 21 (1982) 1263.

[9] R. B. Bird, W. E. Stewart and E. N. Lightfoot, 'Transport Phenomena', Wiley, New York, (1960) pp. 10-14.

[10] G. J. Janz and J. D. E. McIntyre, J. Electrochem. Soc. 109, (1962) 842.

[11] D. S. Newman, R. T. Tillack, D. P. Morgan and W-C. Wan, ibid. 124 (1977) 856.

[12] D. S. Newman and R. M. Stevens, ibid. 131 (1984) 1275.

\footnotetext{
* A Student $t$ test of the data for these melts indicated that the slope of the log shear stress - log shear rate data was statistically different than 1.00 at the $90 \%$ level of significance.
} 